\title{
Study of the Method to Capture the Loose Particles inside the Device Cavity
}

\author{
Xuan Pei*, Shanbin Xi, Zhaofeng Gao and Kui Zhang \\ The $13^{\text {th }}$ Research Institute, CETC, Shijiazhuang 050051, China \\ *Corresponding author
}

\begin{abstract}
The paper introduced the method to capture the particles inside the devices cavity in detail. The method included several steps, which were as follows: particles detection, grinding cover plate, cleaning cover plate, holing cover plate, capturing particles and particles analysis. The particles would be controlled effectively only if the composition and the source could be known well.
\end{abstract}

Keywords-particles; particle impact noise detection (PIND); radiography; scanning electron microscope (SEM)

\section{INTRODUCTION}

During the packaging procedure of the devices, some particles would be left inside the device cavity. Especially, It was hazardable if the particles are conductive. The particles are small-sized and sealed inside the cavity, so the outlines and the materials are unknown. It is necessary to use the appropriate method, so as that the particles can be taken out without new particles bringing inside. According to the GJB 3157-1998, the method was as follows: "Grind the cover plate of the device thinly enough using the polish grinder. Place the cover plate upward and hole it carefully with a edge tool. Cover the hole with the adhesive tape. Put the cover plate downward and shake or knock on it several times, the particles will stick adhere to the adhesive tape." The method did not describe the operating detail of capturing the loose particles. Be based on operating, the paper described the details, including particles detection, grinding cover plate, cleaning cover plate, holing cover plate, capturing particles and particles analysis.

\section{PARTICLES DETECTION}

Generally, two methods are used to detect the particles inside device cavity: Radiography and Particle Impact Noise Detection (PIND)

\section{A. Radiography}

Expose the devices to X-Ray, so the dimension of particles can be known though the radiograph. Usually, according to the location change in two radiographs, particles can be confirmed loose or unloose. But, there is some limitation that only particles with bigger dimension than distinguish ability of the instrument which can block off X-ray can be detected. Sometimes, there still is misjudgment.

Two devices from one batch labeled A and B were detected existing particles in radiography filtrating. Figure 1 and Figure 2 show the images of device A and B radiograph two times. It can be known that particle in device A cavity is loose while unloose in B. Unseal the devices, a metallic spheriform loose particle was found inside device A cavity and nothing in B. But there was some compound which was created by co-reaction between bonding wire and pad at uncertain particle position. The compound was misjudged as the particle in radiography, as shown in Figure 3 and Figure 4.

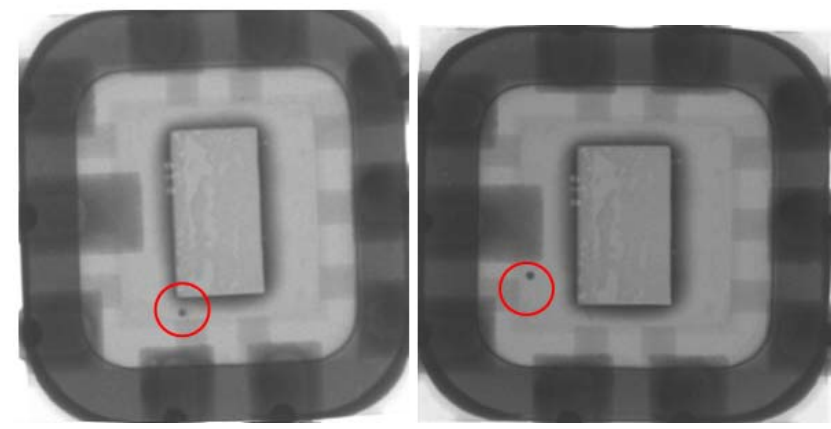

FIGURE I. IMAGES OF DEVICE A RADIOGRAPH TWO TIMES

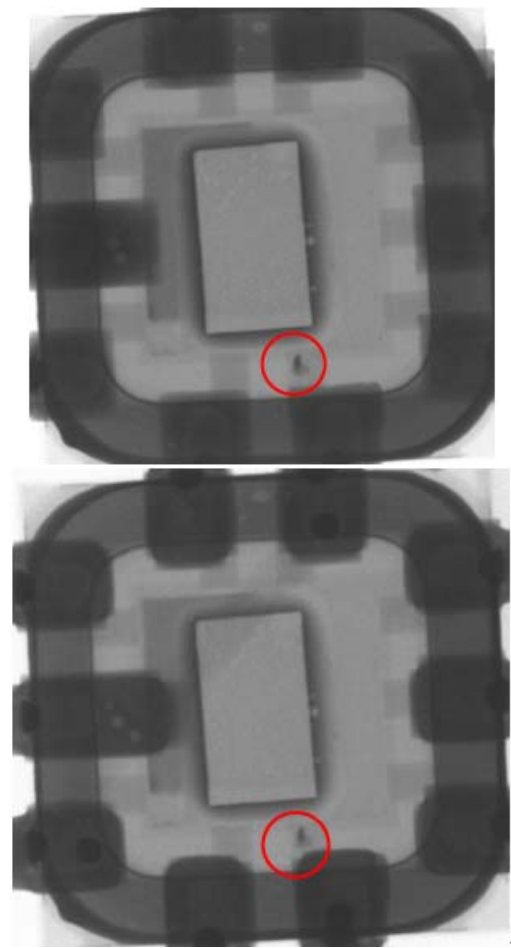

FIGURE II. IMAGES OF DEVICE B RADIOGRAPH TWO TIMES 


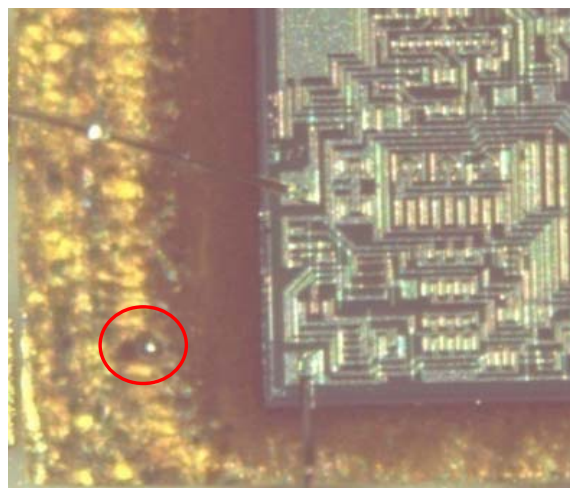

FIGURE III. LOOSE PARTICLE IN CAVITY OF DEVICE A

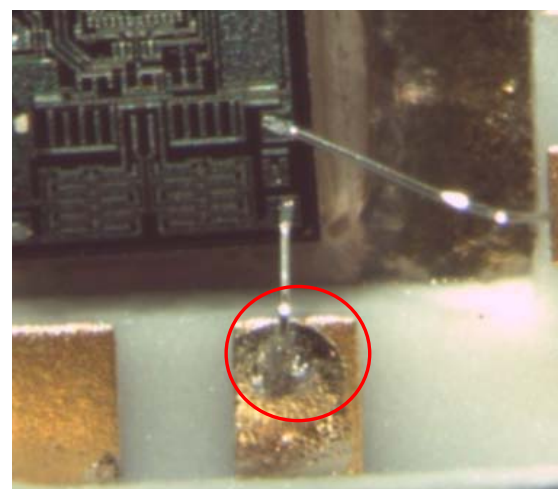

FIGURE IV. EXCEPTIONAL DOT IN CAVITY OF DEVICE B

\section{B. Particle Impact Noise Detection (PIND)}

Impose appropriate shock and vibration on devices, so that loose particles will have some movement and shock. The particles would make noise by striking device cavity. So particles can be detected by transducer capturing noise. Sometimes, because of the structure particularity of some devices, there will be misjudgment.

One device failed to pass the PIND test, but no particle was found inside the cavity. The structure of device cavity without cover plate was as shown in Figure 5. After parallel seam sealing, there was a air gap between cover plate and thick wall of cavity except the welding neck part. The structure could cause resonation in PIND test, and this resulted in PIND failure.

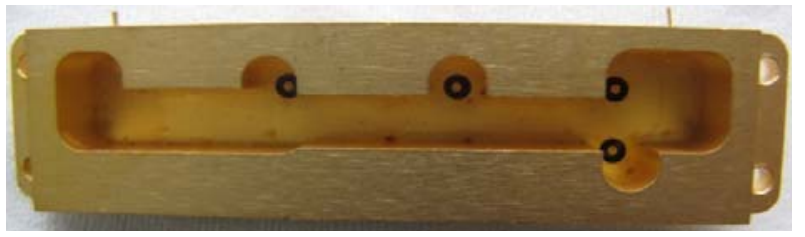

FIGURE V. STRUCTURE IMAGE OF CAVITY

If necessary, PIND can combine with radiography to detect loose particles.

\section{GRINDING COVER PLATE}

There is a problem if you grind the cover plate according to the national standard. Encapsulating was operating in vacuum atmosphere and the device cavity was full with nitrogen, so the cover plate had a trend downward because of atmospheric pressure after encapsulation. During the grinding, as the cover plate getting thinner, the pressure it can withstand would be smaller. Consideration of atmospheric pressure and weight of rag wheel, the deformation would become severe. The deformation would create a force downward on welding neck. The bigger cover plate area is, the severer deformation is, the bigger pull is. The solder in welding neck will be broken by the pull and drop into the cavity as loose particles which intermix with particles born in cavity. The cover plate deformation when grinding is shown in Figure 6. After grinding, hole in the thinnest part of cover plate, that is to say the welding neck part. For the cover plate with large area, particles will be hard to knock out of cavity because the hole is at the corner position.

Holing at the center of cover plate using a appropriate aiguilles can avoid welding neck broking. Also, the particles can be knocked out easily. Figure 7 gave the cover plate appearance after grinding.

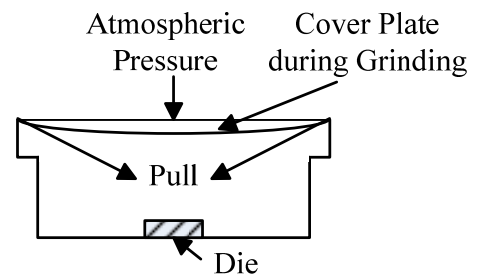

FIGURE VI. SCHEMATIC DIAGRAM OF COVER PLATE DEFORMING

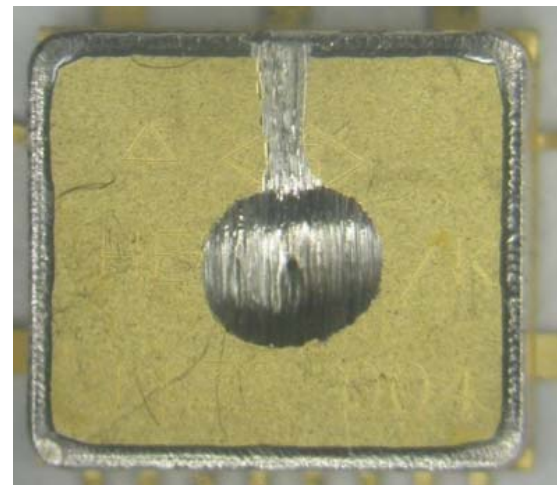

FIGURE VII. APPEARANCE OF COVER PLATE AFTER GRINDING

\section{Cleaning Cover Plate}

Cleaning cover plate is very important to differentiate and analyze particles, but not specified in the national standard. If cover plate cannot be cleaned thoroughly, the particles born in cavity will mix with package fragment. Two kinds of particles stick together adhere to the adhesive tape, which are difficult to distinguish even examined under scanning electronic microscope. After blowing by compressed air and wiping by absolute alcohol the holing part of cover plate, the particles adhered to the adhesive tape are shown in Figure 8. It can be known that the cleaning is not thorough. Stick to the cover plate especially the hole part several times using a tape with strong adhesive, and the improved method is proved to be effective. Figure 9 shows particles adhered to the tape. 


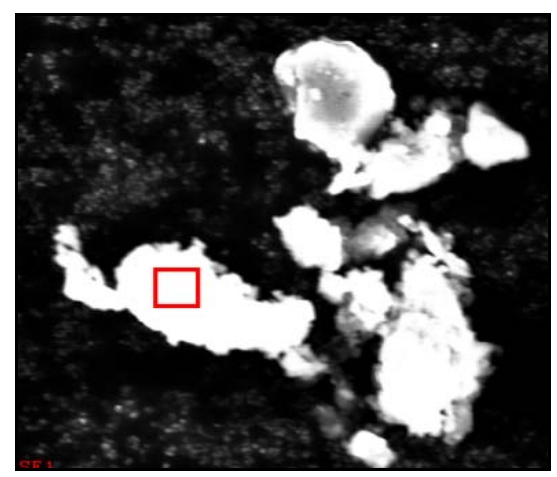

FIGURE VIII. CLEANING BY COMPRESSED AIR AND ALCOHOL SWABS

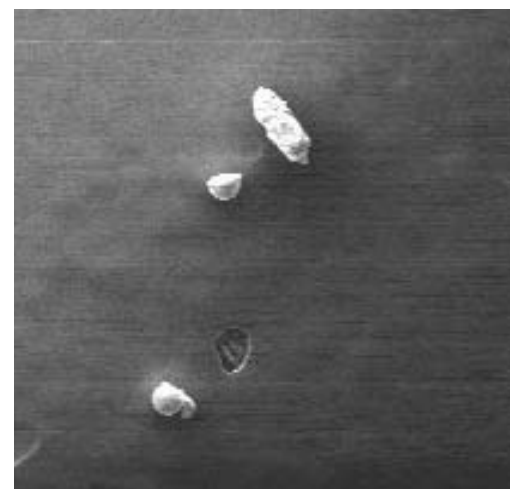

FIGURE IX. CLEANING BY TAPE WITH STRONG ADHESIVE

\section{V.Holing Cover Plate And CAPTURing PARTiCles}

After cleaning the holing part thoroughly, hole it carefully with a edge tool as is mentioned in standard. Cover the hole with the adhesive tape. Put the cover plate downward and shake or knock on it several times. Ordinary tape, like scotch tape, adhesive plaster and so on, it can successfully stick particles, but it will be difficult to find the particles under SEM (to analyze the composition of particles) due to nonconductivity of ordinary tape. Figure 10 and Figure 11 show the optical microscope image and SEM image of particles on ordinary tape, respectively. As is shown in Figure 9, it is easy to find particles on conductive tape in SEM image.

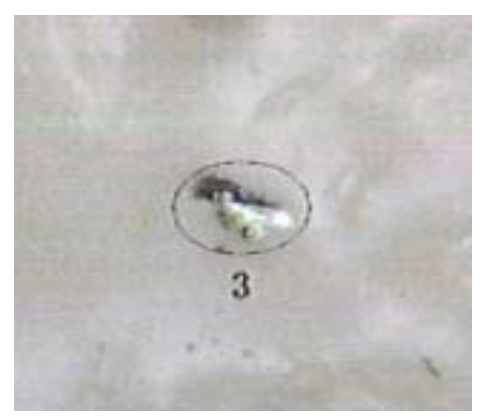

FIGURE X. IMAGES OF PARTICLES UNDER OPTICAL MICROSCOPE

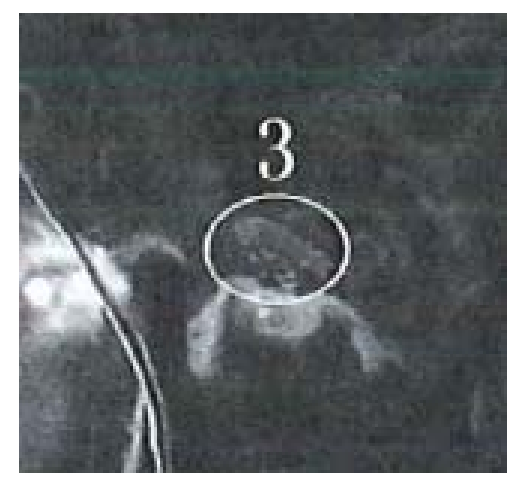

FIGURE XI. IMAGES OF PARTICLES UNDER SEM

\section{PARTICLES ANALYSIS}

Analyze composition of the particles by SEM and find out the resources. Only in this way, the particles can be controlled effectively. Although, cleaning by tape with strong adhesive is a efficient method, it is still difficult to avoid any package fragment dropping into cavity. Therefore, in order to save time and cost, the particles should be differentiate simply from appearance in SEM images. The appearances of particles born in cavity and dropped into cavity during holing are different, as shown in Figure 12 and Figure 13. The former one is almost round and the latter one has obvious grinding trail.

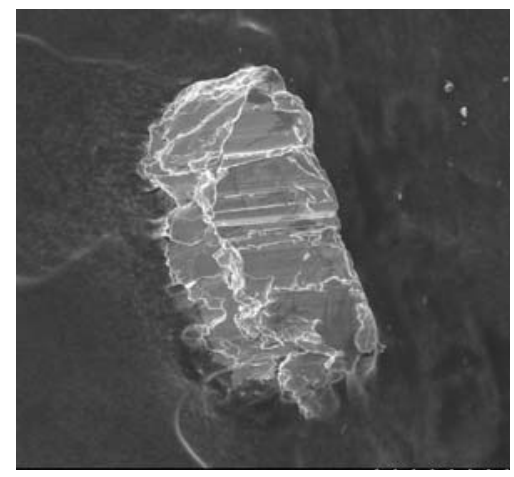

FIGURE XII. IMAGE OF PARTICLE DROPPED INTO CAVITY DURING HOLING

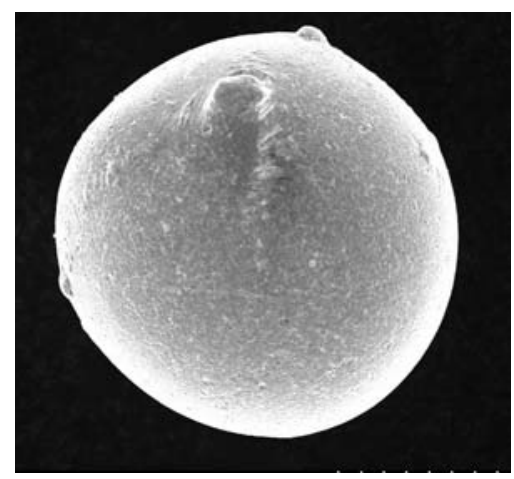

FIGURE XIII. IMAGE OF PARTICLE BORN IN CAVITY

\section{CONCLUSION}

The paper described detecting, capturing and analyzing the particles inside cavity in detail. If the sources of particles are 
known well, the relevant techniques can be proved, so that particles can be controlled at root effectively.

\section{REFERENCES}

[1] Failure Analysis Methods and Procedures for Semiconductor Discrete Devices (Standards Press of China, 1998).

[2] G.H. Zhang, Y. Du, C. Hua, M.H. Huang and H. Cai. Electronic Product Reliability and Environmental Testing Vol. 24(2)(2006), p. 41.

[3] W.L. Zhu, J.H. Gong, J. Lu. Electronics \& Packaging Vol. 4(3)(2004), p. 51.

[4] B. Xu, S.C. Wang, G.F. Zhai. Electromechanical Components Vol. 28(2008), p. 6.

[5] Y.B. Du, X.M. Ling. Semiconductor Technology Vol. 36(2011), p. 247. 